BNL-107110-2014-JA

\title{
Laser Ion Source with Solenoid Field
}

\author{
Takeshi Kanesue, ${ }^{1, a)}$ Yasuhiro Fuwa, ${ }^{2,3}$ Kotaro Kondo, ${ }^{4}$ and Masahiro Okamura ${ }^{1}$ \\ ${ }^{1)}$ Collider-Accelerator Department, Brookhaven National Laboratory, Upton, \\ New York 11973, USA \\ ${ }^{2)}$ Graduate School of Science, Kyoto University, Kitashirakawa, Sakyo, Kyoto 606-7501, \\ Japan \\ 3) RIKEN, 2-1 Hirosawa, Wako, Saitama 351-0198, Japan \\ 4) Research Laboratory for Nuclear Reactors, Tokyo Institute of Technology, \\ 2-12-1 Ookayama, Meguro, Tokyo 152-8550, Japan
}

(Dated: 4 November 2014)

Pulse length extension of highly charged ion beam generated from a laser ion source is experimentally demonstrated. The laser ion source (LIS) has been recognized as one of the most powerful heavy ion source. However it was difficult to provide long pulse beams. By applying a solenoid field $(90 \mathrm{mT}, 1 \mathrm{~m})$ at plasma drifting section, a pulse length of carbon ion beam reached $3.2 \mu$ s which was 4.4 times longer than the width from a conventional LIS. The particle number of carbon ions accelerated by a radio frequency quadrupole (RFQ) linear accelerator was $1.2 \times 10^{11}$, which was provided by a single $1 \mathrm{~J} \mathrm{Nd}$-YAG laser shot. A laser ion source with solenoid field could be used in a next generation heavy ion accelerator.

PACS numbers: 07.77.Ka, 29.20.Ej, 29.27.-a

A performance of a particle accelerator strongly depends on its ion source. An intense particle source may encourage an accelerator concept to become a next generation accelerator project. Especially for heavy ion accelerators, it has been difficult to provide intense beams with highly-charged states. Although there are many operating proton sources with more than a few tens of milliampere in the world $^{1,2}$, there was no project assuming the same amount of current with highly-charged heavy ion beam.

Currently, electron cyclotron resonance ion source (ECRIS) is the most established ion source and commonly used in major accelerator laboratories. Electron beam ion source (EBIS) is also used to provide highly-charged heavy ion beam. Both ion sources typically provide hundreds of microamperes to milliampere range of heavy ions with more than charge state ten ${ }^{3-7}$.

On the other hand, laser ion source (LIS) can provide highly-charged ion beam with a beam current of more than hundreds of milliamperes. However, it has never been adopted by major stream of the accelerator's history since it was proposed in $1969^{8,9}$ because of mainly three problems: beam transportation, stability, and short beam pulse.

A LIS uses a pulsed high power laser focused onto a solid state target. The laser is absorbed in the target, and target temperature goes up above transition temperature from solid to vapor and plasma in the range of $10^{12} \mathrm{~W} / \mathrm{cm}^{2}$ of laser power density. Electrons in the plasma are thermalized by absorbing laser photons by inverse bremsstrahlung process, and ionization proceeds. Initial plasma density produced by laser ablation is much higher than that of ECRIS and EBIS. At the same time as plasma production, the laser-produced plasma expands to perpendicular to the target surface ${ }^{10}$. Also, plasma adiabatically expands to both transverse and longitudinal direction. This expansion makes ion beam pulse from nanosecond-range to microsecond-range. Current density and pulse width of the plasma follow the relationships below during expansion:

$$
\begin{array}{r}
T \propto L \\
\rho \propto L^{-3}
\end{array}
$$

a)Electronic mail: tkanesue@bnl.gov 

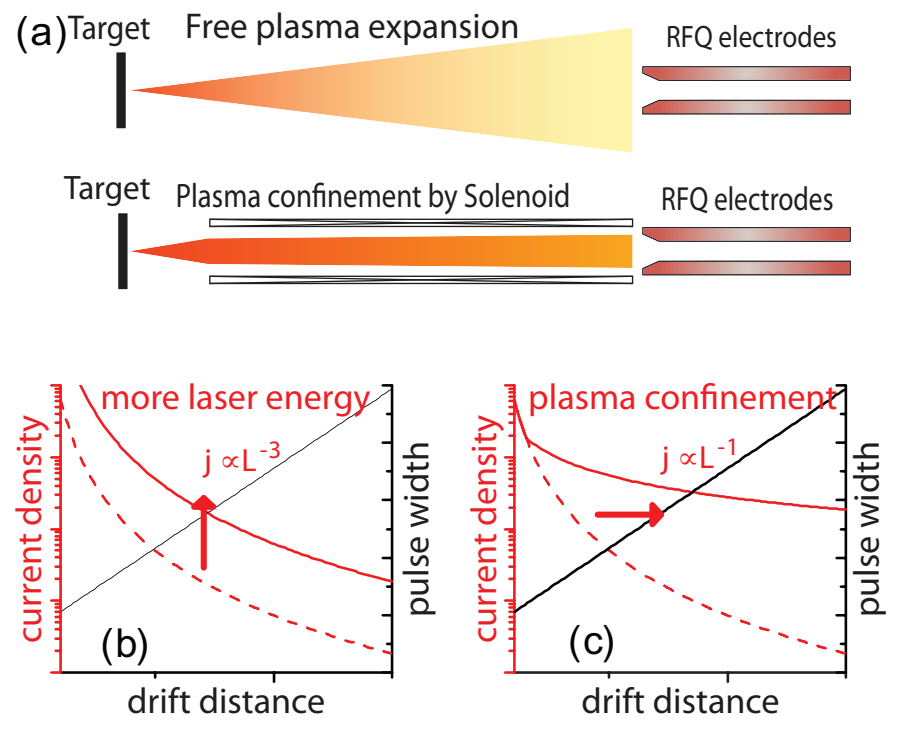

FIG. 1. (color online) Top and bottom of (a) are a conventional scheme and a scheme with solenoid, respectively. (b) shows the conventional scheme to extend pulse width. (c) describes the concept with a solenoid field.

where, $T, \rho$, and $L$ are pulse width, ion current density at an ion extraction point, plasma drift distance which is the length between the target and the ion extraction point, respectively. In case short pulse width is not a problem, we can achieve more than hundreds of milliamperes of ion beam by shortening the plasma drift distance.

However, it was difficult to transport and inject such an intense heavy ion beam to a first stage linear accelerator, typically radio frequency quadrupole (RFQ) linac because of a space charge effect. At CERN, as a part of LHC project, a high power LIS using 100 $\mathrm{J} \mathrm{CO}_{2}$ laser had been studied intensively under the collaboration with ITEP ${ }^{11}$, but was terminated in 2003 due to low transmission efficiency through the RFQ and stability issue.

The beam transportation problem was completely overcome by a direct plasma injection scheme (DPIS) proposed in $2000^{12}$. In this scheme, ions are transported in neutralized plasma state to avoid beam loss issue at low energy beam transport line (LEBT). It was verified experimentally that more than $10 \mathrm{~mA}$ of fully stripped carbon beam can be accelerated with this scheme ${ }^{13,14}$. Also a series of experiments showed that good beam stability can be achieved by precise control of a target position during experiment and use of a stable solid state laser system. However, the obtained pulse length was less than $1 \mu \mathrm{s}$. A short pulse length was the last issue to be solved in the LIS.

The cause of short pulse is free expansion to all direction during plasma transportation. As drifting, current density rapidly decreases proportionally to $L^{-3}$, so there is a trade-off between current and pulse width.

To extend ion beam pulse width, we adopt a transverse confinement of plasma by a solenoid magnet. Although researchers have been investigated the effect of an axial magnetic field on plasma, their primary concern is to increase a current density by focusing the plasma ${ }^{15}$ or to bend a plasma transport line to separate impurities ${ }^{16}$. However, our purpose is to transport laser-produced plasma for a long distance to extend the plasma longitudinally. Solenoid magnet is installed in a plasma expanding line. The solenoid is expected to depress the plasma free expansion to transverse direction because plasma with ion and electron moves along magnetic field. The plasma expands only to longitudinal direction as same as the free expansion without a solenoid magnet.

In the conventional LIS, to extend the beam pulse width we need to increase initial amount of plasma by increasing laser power. A use of a solenoid magnet provides alternate scheme to extend the pulse width. This concept is compared with the conventional scheme 


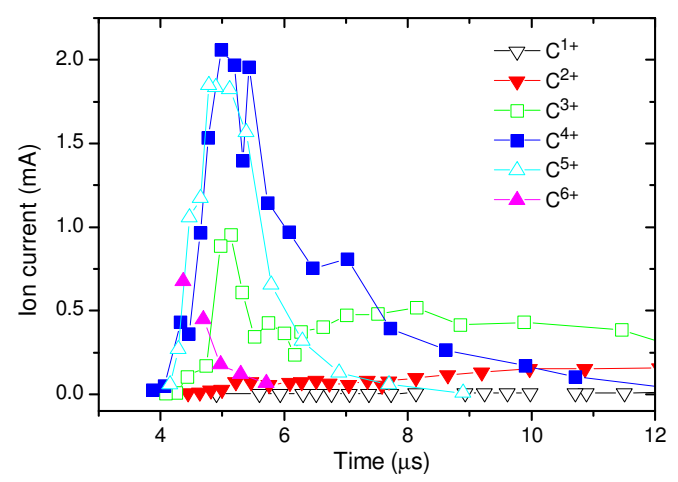

FIG. 2. (color online) Charge state distribution in a carbon plasma at ion extraction point.

in Fig. 1. The plasma density is enhanced through the solenoid and the long drift distance is achieved without steep dilution. Transportation of neutralized plasma without space charge in a solenoid magnet with high current density can overcome the last remained issue of laser ion source.

In this letter, we describe the LIS concept using solenoid field as a guide of laser-produced plasma, and the experimental result of the LIS combining DPIS with solenoid magnet. A RFQ accelerator dedicated to DPIS ${ }^{17}$ was used. We obtained $36 \mathrm{~mA}$ of $\mathrm{C}^{4+}$ beam accelerated to $100 \mathrm{keV} / \mathrm{u}$ by the RFQ with pulse width of $3.2 \mu \mathrm{s}$. The pulse width was increased by a factor of four based on the previous experiment. We verified that the LIS with solenoid could overcome the short pulse issue.

Prior to an acceleration test, charge state distribution in plasma was measured to estimate the ion beam properties without a solenoid magnet. The experiments have been carried out with a $1 \mathrm{~J} / 6 \mathrm{~ns}$ Nd:YAG laser with $1064 \mathrm{~nm}$ wavelength. The setup and analysis method is similar to those used in earlier experiment ${ }^{18}$.

A laser light with an incident angle of 30 degrees to the target, was focused onto a solid target by a convex lens $\left(\mathrm{f}=100 \mathrm{~mm}\right.$ ). The estimated laser power density was $6 \times 10^{12}$ $\left[\mathrm{W} / \mathrm{cm}^{2}\right]$. Carbon target was perpendicularly-placed to the beam line in the chamber. The target position was changed after every shot to provide a fresh surface. The residual gas pressure in entire setup was kept bellow an order of $10^{-4} \mathrm{~Pa}$ to reduce recombination processes of the ablation plasma.

Total ion current was measured by a Faraday Cup (FC) at a distance of $2.38 \mathrm{~m}$ from the target. The FC had a suppressor mesh biased to $-3.5 \mathrm{kV}$ to separate ions from plasma. To measure charge state distribution, an electrostatic ion analyzer (EIA) was used instead of the FC. A secondary electron multiplier (SEM) (Hamamatsu R2362) biased to $-3.5 \mathrm{kV}$ at a distance of $3.70 \mathrm{~m}$ from the target detected ions after the EIA. The trigger signal was set by a photo detector detecting the laser shot. A time-of-flight (TOF) spectra of each charge state were obtained by scanning the different EIA voltages.

The TOF spectra were calibrated by total current of the FC signal assuming a gain of the SEM was independent to ion energy and charge states. The achieved spectrum were converted based on Eqs. (1) and (2) to the extraction condition for the following ion-acceleration experiment (1.33 $\mathrm{m}$ from the target with $6 \mathrm{~mm}$ extraction aperture).

Figure 2 shows the TOF spectrum at the extraction point. We care mainly charge state more than four because an ion source and a RFQ were set to maximize a $\mathrm{C}^{4+}$ yield in the following acceleration test. The maximum ion current within the $6 \mathrm{~mm}$ aperture is 2.1 $\mathrm{mA}$ of $\mathrm{C}^{4+}$ with pulse width of $1.3 \mu \mathrm{s}$ at full width at half maximum (FWHM). Ions of charge state over four reach the extraction point at the time between 4 to $10 \mu$ s while ions of charges state less than three have longer tails.

After charge state distribution was measured, we then studied a pulse extension of laser- 


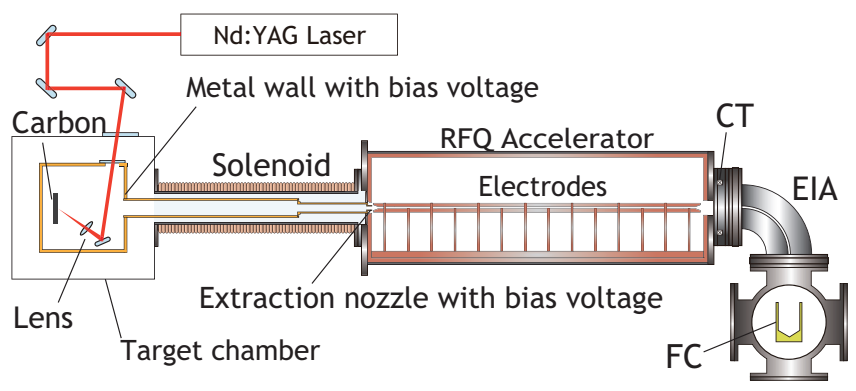

FIG. 3. (color online) Setup for ion acceleration by RFQ.

produced plasma by the solenoid magnet. The experimental setup is shown in Fig. 3. This $\mathrm{RFQ}$ can accelerate ion beam with a charge-to-mass ratio more than $1 / 3$ corresponding to $\mathrm{C}^{4+}$ from $20 \mathrm{keV} / \mathrm{u}$ to $100 \mathrm{keV} / \mathrm{u}$. More details of the RFQ are shown in other paper ${ }^{17}$. The laser irradiation condition was same as those in the plasma measurement. The target plate was placed in an isolated plasma production chamber located inside a target chamber. The target was connected to the 3D manipulator through an isolated ceramic bar. The target and the plasma production chamber were electrically connected and biased to $60 \mathrm{kV}$ to match the RFQ injection energy of $20 \mathrm{keV} / \mathrm{u}$ for $\mathrm{C}^{4+}$.

Inside of the plasma production chamber and solenoid, the plasma expanding volume was surrounded by metal walls to keep same electric potential between the target and extraction point. Ions were extracted between the nozzle, which had $6 \mathrm{~mm}$ inner diameter, and RFQ electrodes at a distance of $1.33 \mathrm{~m}$ from the target. A 1-m-long solenoid magnet was installed between the target chamber and the RFQ. The maximum magnetic field of the solenoid was $90 \mathrm{mT}$. The distance between the target and the solenoid was $375 \mathrm{~mm}$ and a distance between the solenoid and the extraction point was $80 \mathrm{~mm}$.

A current profile was measured by a Current Transformer (CT) at the exit of the RFQ. Ion beam after RFQ was analyzed by the EIA again with FC instead of the SEM. The FC had suppressor mesh biased to $-0.5 \mathrm{kV}$.

The CT detects a total ion beam profile. To discuss contents of the beam from the RFQ, we define three types of ion beam out of the RFQ. The first group is fully accelerated beam, which is a beam accelerated to the RFQ nominal output energy. The second group is partially accelerated beam, which is a beam dropped from acceleration condition of the RFQ during acceleration and not accelerated to the nominal energy. The third group is an unaccelerated beam, which is a beam not accelerated by the RFQ but trapped and transported by the RFQ transverse field to the exit.

The accelerated beam was sampled by the slit and was analyzed by the EIA. By scanning the applied voltage to the EIA, the fully accelerated beam of $\mathrm{C}^{4+}, \mathrm{C}^{5+}$, partially accelerated beam, and the unaccelerated beam could be extracted.

To reconstruct the unaccelerated beam, signals measured by the FC at the EIA voltages corresponding to the unaccelerated beam were divided by EIA voltages and summed considering a EIA voltage step. This reconstructed beam profile was normalized by a tail of the CT signal where no accelerated signal was observed. Figure 4 shows the CT signal and the accelerated beam profile. The CT peak current was recorded at $45 \mathrm{~mA}$. The unaccelerated beam was observed in the slow part of the CT signal, so the main part of the CT profile consisted of the fully and partially accelerated beam.

The rest of the beam after subtracting the unaccelerated beam from the CT signal is the accelerated beam. We estimated ion yield of fully accelerated $\mathrm{C}^{4+}$ beam using obtained fractions of each charge state. We assumed the same transmission from CT to FC for all ions, though ion beam with lower energy and higher current beam can have larger diverging angle and lower transmission due to space charge effect. This leads to underestimation of higher energy ion yield.

The charge ratio of fully accelerated $\mathrm{C}^{4+}, \mathrm{C}^{5+}, \mathrm{C}^{6+}$, and partially accelerated beam were 


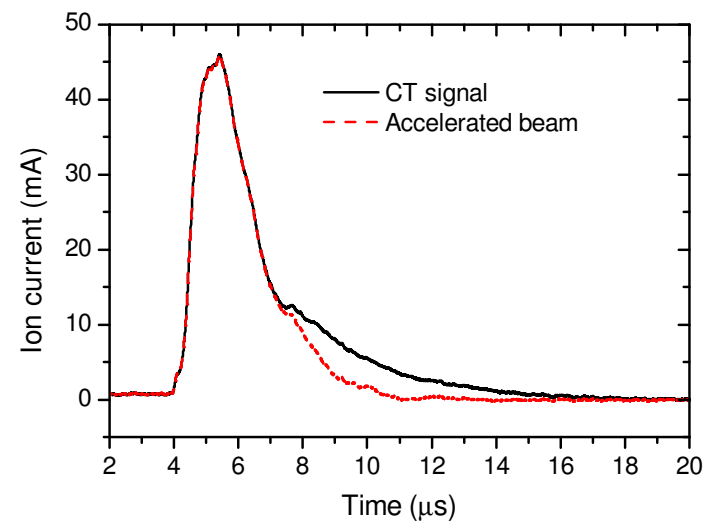

FIG. 4. (color online) Carbon beam measured by CT at the exit of RFQ and wave form without unaccelerated beam, respectively.

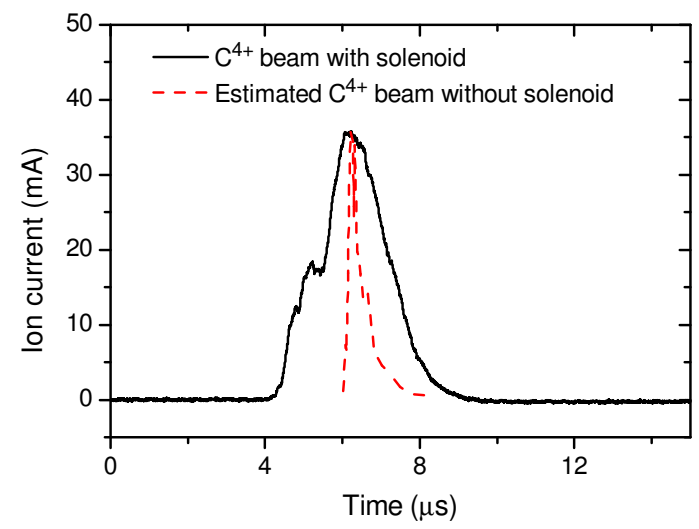

FIG. 5. (color online) $\mathrm{C}^{4+}$ beam with and without solenoid under the same laser irradiation condition. The wave form without solenoid is estimated based on the plasma measurement.

$87 \%, 9.6 \%, 1.3 \%$, and $2.2 \%$, respectively. Based on this ratio, the number of the fully accelerated particles of $\mathrm{C}^{4+}, \mathrm{C}^{5+}, \mathrm{C}^{6+}$ were estimated to be $1.2 \times 10^{11}, 1.0 \times 10^{10}$, and $1.2 \times 10^{9}$, respectively. The pulse width at $20 \%$ of maximum current of plasma at the RFQ injection point was $3.4 \mu \mathrm{s}$, and that of accelerated beam was $3.2 \mu \mathrm{s}$. Ion beam induced from laser ablation commonly has long tail. To truncate the tail, we define pulse width at $20 \%$ of maximum current.

Figure 5 shows wave forms of $\mathrm{C}^{4+}$ beam with and without solenoid under the same laser irradiation condition. The solid line represents the accelerated beam with solenoid field reconstructed from the analyzed data. The dotted line is the $\mathrm{C}^{4+}$ beam without solenoid field. It is estimated based on the plasma measurement shown above. To obtain same peak current, $36 \mathrm{~mA}$, a plasma drift length can be calculated as $0.29 \mathrm{~m}$. Pulse length of the solid line was $3.2 \mu \mathrm{s}$, which is 4.4 times longer than that of the dotted line, $0.7 \mu \mathrm{s}$. Since the plasma drift length with the solenoid result was $1.33 \mathrm{~m}$, this extension factor agrees with the ratio of plasma drift lengths.

The third set of the experiment were carried out to investigate the effect of solenoid field strength to the plasma guide. The same setup as the previous experiment was used. Ion beam analysis using the EIA showed that the time structure and the fraction of each ion beam group were similar to those in the previous experiment with $90 \mathrm{mT}$ solenoid field. Figure 6 shows the peak current and the pulse width at FWHM of the CT signal as a 


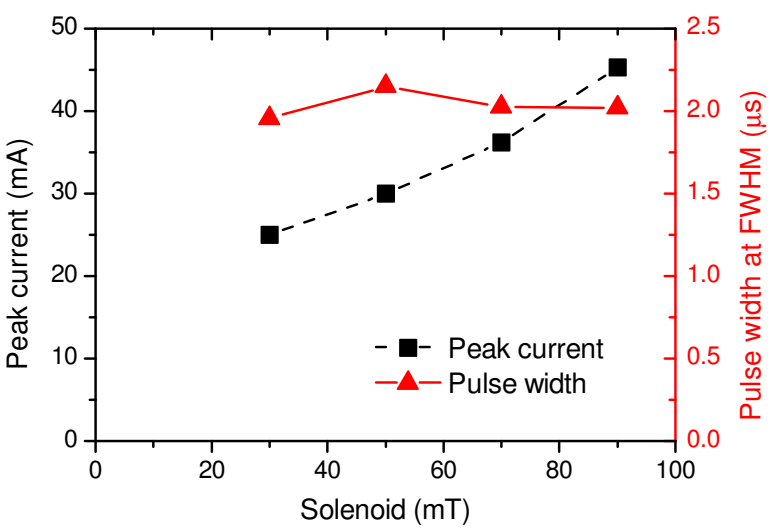

FIG. 6. (color online) CT peak current and pulse width at FWHM as a function of solenoid field.

function of the solenoid field. The accelerated beam current was almost linearly increased to the solenoid field and not saturated at $90 \mathrm{mT}$ of the maximum solenoid field. The solenoid field was limited to $90 \mathrm{mT}$ due to our setup restriction. It was found that a solenoid can be used as a control knob for the accelerated current using a LIS.

In conclusion, we verified that the extension of pulse width of the extracted beam from the LIS by transporting the laser produced plasma in the solenoid magnet. Also, we showed that the confinement of the laser produced plasma can be controlled by the solenoid field. These conclusions correspond to the plot shown in Fig. 1, That is, a current density is no longer follow Eq. (2). Because the limitation on a pulse width was overcome, the use of LIS should be considered to provide much intense beam than that from the existing ion sources.

This work was supported by U.S. Department of Energy, RIKEN, and Japan Society for Promotion of Science.

${ }^{1}$ R. F. Welton, J. Carmichael, N. J. Desai, R. Fuga, R. H. Goulding, B. Han, Y. Kang, S. W. Lee, S. N. Murray, T. Pennisi et al, Rev. Sci. Instrum. 81, 02A727 (2010).

${ }^{2}$ H. Oguri, A. Ueno, K. Ikegami, Y. Namekawa, and K. Ohkoshi, Phys. Rev. ST Accel. Beams 12, 010401 (2009).

${ }^{3}$ C. Lyneis, D. Leitner, M. Leitner, C. Taylor, and S. Abbott, Rev. Sci. Instrum. 81, 02A201 (2010).

${ }^{4}$ L. T. Sun, H. W. Zhao, W. Lu, X. Z. Zhang, Y. C. Feng, J. Y. Li, Y. Cao, X. H. Guo, H. Y. Ma, H. Y. Zhao et al, Rev. Sci. Instrum. 81, 02A318 (2010).

${ }^{5}$ T. Nakagawa, Y. Higurashi, J. Ohnishi, T. Aihara, M. Tamura, A. Uchiyama, H. Okuno, K. Kusaka, M. Kidera, E. Ikezawa et al, Rev. Sci. Instrum. 81, 02A320 (2010).

${ }^{6}$ J. G. Alessi, D. Barton, E. Beebe, S. Bellavia, O. Gould, A. Kponou, R. Lambiase, R. Lockey, A. McNerney, M. Mapes et al, Rev. Sci. Instrum. 81, 02A509 (2010).

${ }^{7}$ E. N. Beebe, J. G. Alessi, O. Gould, D. Graham, A. Kponou, A. Pikin, K. Prelec and J. Ritter, Rev. Sci. Instrum. 73, 699 (2002).

${ }^{8}$ Y. A. Byckovsky, V. F. Eliseev, Y. P. Kozyrev, and S. M. Silnov, "Laser generator of multi-charged ions for accelerators," Sov Patent 324938

${ }^{9}$ N. J. Peacock and R. S. Pease, J. Phys. D 2, 1705 (1969).

${ }^{10}$ B. Toftmann, J. Schou, T. N. Hansen, and J. G. Lunney, Phys. Rev. Lett. 843998 (2000).

${ }^{11}$ B. Sharkov and R. Scrivens, IEEE Transcations on Plasma Science. 33, 1778 (2005).

${ }^{12}$ M. Okamura, T. Takeuchi, T. Katayama and K. Sawada, Proceedings of the 2000 European Particle Accelerator Conference, Vienna, Austria, 26-30 June 2000, p848 (2000).

${ }^{13}$ H. Kashiwagi, M. Fukuda, M. Okamura, R. A. Jameson, T. Hattori, N. Hayashizaki, K. Sakakibara, J. Takano, K. Yamamoto, Y. Iwata and T. Fujimoto, Rev. Sci. Instrum. 77, 03 B305 (2006).

${ }^{14}$ M. Okamura, H. Kashiwagi, K. Sakakibara, J. Takano, T. Hattori, N. Hayashizaki, R. A. Jameson and K. Yamamoto, Rev. Sci. Instrum. 77, 03B303 (2006).

${ }^{15}$ Wołowski, J. Badziak, I. Ivanova-Stanik, P. Parys, W. Stȩpniewski and E. Woryna, Rev. Sci. Instrum. 75, 1353 (2009).

${ }^{16}$ Y. Y. Tsui, D.Vick, and R. Fedosejevs, Appl. Phys. Lett. 70, 1953 (1997).

${ }^{17}$ M. Okamura, K. Katayama, R. A. Jameson, T. Takeuchi, T. Hattori and H. Kashiwagi, Laser and Particle Beams 20, 451 (2002).

${ }^{18}$ S. Kondrashev, T. Kanesue, M. Okamura, and K. Sakakibara, J. Appl. Phys. 100, 103301 (2006). 